\title{
Analysis of a First Professional Year Student Wellness Program
}

Colleen M Lewellyan, PharmD/MBA Candidate; Allison C Bouwma, PharmD Candidate; Lisa A Salvati, PharmD, BCACP; David R Bright, PharmD, BCACP; Minji Sohn, PhD

Ferris State University College of Pharmacy

Author Contributions: Colleen Lewellyan and Allison Bouwma have equal contributions as first author. Lisa Salvati has contribution as senior/corresponding author.

Disclosures: None

\begin{abstract}
Objective: To identify wellness-related needs and assess the impact of wellness-related offerings among first professional year pharmacy students.

Innovation: A survey tool was developed and offered to P1 students at the beginning and end of their fall and spring semesters. Additional biometric data was also collected to help identify wellness needs. Data from the first academic year (AY1) was used to develop targeted wellness interventions offered to $P 1$ students during the subsequent academic year (AY2). Assessment strategies from AY1 were repeated with minor modifications in AY2 to identify changes in baseline needs and changes in markers across the academic year.

Critical Analysis: AY1 survey response rates varied from $20.1 \%$ to $47.4 \%$ across the semester. Frequent dissatisfaction was reported with diet, weight, and exercise. AY2 survey response rates varied from $15.8 \%$ to $58.3 \%$ across the semester. The AY2 cohort demonstrated similar dissatisfaction data; however, also demonstrated lower baseline stress scores as compared to the AY1 cohort, higher baseline BMI, and higher systolic and diastolic blood pressure. Individual interventions offered to AY 2 students were attended by as many as $16.5 \%$ of the academic cohort. Nutrition classes exhibited stronger attendance than fitness classes.

Next Steps: The process used in this study was easily implemented and provided understanding of wellness gaps, which helped to identify interventions that were implemented and assessed. The process also demonstrated that wellness needs can vary from one population to another, reinforcing the value of periodic assessment to identify changing needs.
\end{abstract}

Keywords: student wellness, pharmacy education, nutrition, student stress, exercise

\section{Description of the Problem}

Healthcare professional students have strong healthy lifestyle beliefs, but they lack healthy behaviors. ${ }^{1}$ According to a survey conducted at seven different health professional colleges, over $50 \%$ of participants believed that they were in "very good" or "excellent" health. ${ }^{1}$ However, approximately $40 \%$ of the sample was overweight or obese, $73 \%$ slept for less than 7 hours per night, only $44 \%$ met the recommended weekly exercise goals of at least 150 minutes of moderate aerobic activity per week, and only $32 \%$ reported eating at least 5 servings of fruit and vegetables per day. ${ }^{1}$ Thus, there is a need among healthcare professional students to improve healthy behavior, specifically when it comes to weight, physical activity, sleeping habits, and food choices. Evidence suggests that many medical students sacrifice their physical and psychological well-being for the sake of maintaining good grades, which is a major reason why medical students are at an increased risk for physical and psychological problems. ${ }^{1-4}$

Corresponding author: Lisa Salvati, PharmD, BCACP

Department of Pharmacy Practice, College of Pharmacy, Ferris State University; Email: lisasalvati@ferris.edu
Such a risk is likely to apply to students in other health professions as well. ${ }^{2}$

Wellness interventions can help encourage positive health changes, specifically improving mental and physical health. ${ }^{5-7}$ Wellness programs have been studied in medical students, focusing on stress reduction and personal wellness habits. ${ }^{5}$ One study showed that medical students participating in the program had decreased depression and anxiety scores compared to students who did not participate. ${ }^{5}$ Additionally, aerobic fitness may improve academic performance and have lasting effects on mental health well into cognitive maturity. ${ }^{7}$ These targeted wellness interventions focused on specific populations and demonstrated improvement in wellness endpoints, suggesting that strategic wellness initiatives could be designed for populations with specific wellness needs. While it seems reasonable to assume that there are pharmacy students that do not have healthy lifestyle habits, we are not aware of peerreviewed reports that demonstrate successful targeted wellness programming for pharmacy students. 


\section{Statement of Innovation}

In order to provide targeted wellness support for first professional year pharmacy (P1) students, a process of assessing wellness-related needs among P1 students was developed. The resulting needs assessment data was used in the following academic year to implement and evaluate a series of wellness initiatives.

\section{The Innovation}

Ferris State University (FSU) College of Pharmacy operates on two campuses, with $\mathrm{P} 1$ and $\mathrm{P} 2$ students on a rural, Midwestern campus, and P3 students at an urban medical center. At the time of the study, there were about 140-150 students enrolled per class. During the academic year, the fall semester starts at the end of August and concludes in the middle of December; the spring semester starts at the beginning of January and concludes in the beginning of May. In order to better understand wellnessrelated needs, a survey tool was developed and offered to P1 students at the beginning and end of their fall semester and end of their spring semester. A shortened version of the survey was given at the beginning of the spring semester to assess stress, diet, and weight over the winter break. Biometric assessment (i.e. height, weight, and blood pressure) was also provided at the beginning and end of the fall semester, as well as the end of the spring semester. Biometric data was not obtained at the beginning of the spring semester as the duration of time between the end of the fall semester and the beginning of the spring semester was relatively brief; therefore, there was a reduced chance of observing profound changes in endpoints. Figure 1 outlines the timeline and process that was utilized.

Survey questions were created to assess markers of wellness, namely sleep, diet, exercise, and perception of illness (Supplemental Files 1\&2). The Cohen Perceived Stress Scale ${ }^{8}$ was included to assess stress given the clear relationship between stress and wellness. ${ }^{3}$ During the 2015-16 academic year (hereafter, AY1), a QuestionPro @ survey link (QuestionPro Inc.; San Mateo, CA) was distributed to P1 students the same day that the biometric assessment was offered. Biometric assessments were offered during a class break within the pharmacy building for student convenience; lunch was provided to participants.

Data from AY1 were used by the researchers to help identify potential wellness interventions that could be offered during the subsequent academic year (2016-17, hereafter AY2). Specific findings from AY1 included high stress levels among students, lack of knowledge related to caloric intake, and lack of exercise. Students also reported high levels of dissatisfaction with their current diet, exercise, weight, sleep, and stress level. In response to these findings, the researchers developed a series of interventions to impact nutrition and exercise, which would potentially also impact stress.
The exercise interventions consisted of yoga, Zumba, ${ }^{\circledR}$ and strength training. Each exercise intervention was offered once a week after classes were completed for the day in the pharmacy building for student convenience. The nutrition interventions were focused classes offered during the lunch hour, which occurred approximately once per month. These interventions were chosen based on availability, cost, and feasibility. Snacks were provided, and prizes were raffled off at each nutrition and exercise class to encourage participation. Exercise equipment, snacks, instructor stipends, and prizes were funded through alumni and College support.

The survey tool used for baseline assessment in AY1 was used in AY2 to identify any deviations in the needs assessment and/or biometric data, as well as to assess changes in responses throughout the semester following the implementation of the wellness interventions. Slight modifications to the assessment strategy were made in AY2 based on lessons learned in AY1. This included capturing body fat percentage data during the biometric evaluation and separating the biometric data collection from the survey; the survey was open for a week to improve response rates. The needs assessment study and the intervention study were approved by the FSU Institutional Review Board. AY1 ordinal data was analyzed using the KruskalWallis test while continuous data was analyzed using ANOVA. When comparing AY1 and AY2, ordinal data was analyzed using the Mann-Whitney test and continuous data was analyzed using a t-test. Data was analyzed using Stata $13^{\circledR}$ (StataCorp LLC; College Station, TX).

\section{Critical Analysis}

Among students in the AY1 cohort, survey response rates at the four time points during the year ranged from $20.1 \%$ to $47.4 \%$. Frequent dissatisfaction was reported with diet, weight, and exercise (48.1\%, 53.9\%, and $75.1 \%$, respectively). In the final assessment point of AY1, greater than $50 \%$ of students reported disagreeing or strongly disagreeing to being satisfied with each of their current exercise, diet, weight, quality of sleep, and stress level. Stress increased during each semester, and higher stress was correlated with higher body mass index scores. Additionally, approximately $37 \%$ of AY1 students reported not knowing how many calories they had consumed in the last 24 hours. Students were reporting less than 8 hours of sleep per night during the week and approximately 9 hours on the weekends. The total time spent napping decreased throughout the academic year. Greater than $85 \%$ of students reported spending less than 60 minutes per week engaging in moderate to vigorous strength training exercises and greater than $65 \%$ reported less than 60 minutes per week engaging in moderate to vigorous cardiovascular exercise. 
Figure 1: Study Timeline for First Year Pharmacy Student Wellness Initiatives

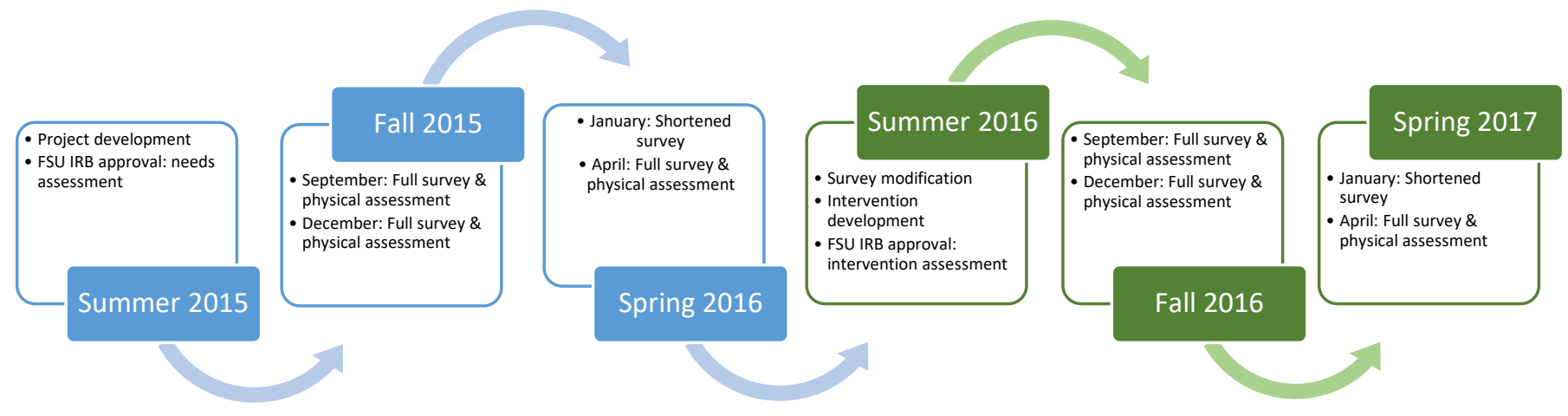

The AY2 cohort demonstrated similar response rates (15.8\% to $58.3 \%)$, and dissatisfaction data was also generally similar. However, students in the AY2 cohort demonstrated lower baseline stress scores as compared to the AY1 cohort (14.1 vs. 16.7, $p=0.028)$, higher baseline BMI (26.2 vs. 23.9, $p=0.034)$, higher systolic (129.6 $\mathrm{mmHg}$ vs. $120.5 \mathrm{mmHg}, \mathrm{p}=0.002)$ and diastolic blood pressure ( $82.5 \mathrm{mmHg}$ vs. $75.7 \mathrm{mmHg}, \mathrm{p}=0.004)$, and increased weeknight sleep ( 8.4 hours vs. 7.4 hours, $\mathrm{p}<0.001)$. Across AY2, dissatisfaction with stress increased on average from $34.0 \%$ at the beginning of each semester to $62.7 \%$ at the end of each semester. Individual intervention sessions offered to AY2 students were attended by as many as $16.5 \%$ of the P1 student cohort. Nutrition classes exhibited stronger attendance (13 participants on average) than fitness classes (2 participants on average). According to the survey data, AY2 students who participated in the nutritional interventions and stated they incorporated the knowledge gained reported eating out fewer times per week (1.4 vs. 2.3, $p<0.001)$, consuming less servings of fat (1.3 vs. $2.2, p=0.042)$, and consuming more servings of fruit ( 2.3 vs. $1.3, p=0.033)$.

\section{Key Findings}

This study describes the process to identify wellness-related needs among P1 students, and then develop and test a series of interventions. The data from the needs assessment in AY1 demonstrated gaps in student wellness that could be positively impacted by providing wellness-related interventions for P1 students. The wellness interventions were assessed for both student perceptions of wellness and any associated health outcomes improvement over the course of the study. It was specifically apparent that assistance could be provided in the areas of diet, exercise, and stress.

Based on the experience of managing the implementation of wellness-focused interventions for P1 students, the authors offer several considerations for readers with similar interests. A key learning point from this study was that student needs can vary within the same class and between each academic year. A way to address this issue when implementing wellnessrelated programs is to determine the baseline needs of the class and tailor strategies to accommodate those needs. A needs assessment is a feasible way to determine the areas of wellness that students feel would provide them the most benefit. It may also be helpful to repeat a needs assessment periodically during the academic year (e.g. once or twice) to determine changes in needs over time.

Some of the challenges we experienced were related to the number of students who participated in the interventions. We anticipated that attendance at the various intervention sessions would likely be our biggest challenge, especially considering that participation in worksite-related wellness programs is documented to achieve less than $50 \%$ of employee participation on average. ${ }^{9}$ In this study, $16.5 \%$ was the largest participation rate during a single intervention session. However, as each student presumably had unique wellnessrelated needs, it is unexpected for the same students to take part in such a wide variety of wellness offerings. Therefore, it is likely that greater than $16.5 \%$ of students took part in at least one wellness intervention at some point throughout the year. It was difficult to determine if the students who utilized the interventions were the ones who would benefit the most from these interventions as no data was collected on the students who did not participate in any of the surveys or interventions. Some strategies for improving participation include offering the classes free of charge to the students to ease financial burden, as well as keeping convenience in mind by having interventions take place after classes or during breaks to ensure that students will have minimal school-related conflicts. Being mindful of exam schedules and knowing that low-stress periods are when students will have more time to focus on their health can help the success of wellness-related programs. Encouraging participation in healthy habits outside of school should become a routine part of a program. 
Additionally, campus resources for students, such as nutritional counseling and intramural sports, could be publicized to provide students with many options. Further investigation into the types of interventions that would be most attractive to student participants may be helpful for related program development and expansion. Early determination of student needs and flexibility in program offerings can better detect worthwhile interventions.

Lastly, this study was conducted at a Midwest University's College of Pharmacy so further research is needed to determine whether needs and successful interventions are different in other geographical areas as well as other disciplines.

\section{Next Steps}

Pharmacy student wellness is an area with wide literature gaps that lacks an established process to identify wellness needs and lacks information related to wellness intervention development. The process used in this study proved to be easily implemented and provided an understanding of wellness gaps. The wellness gaps identified were then utilized to guide intervention development for P1 students. Others could use the process described to identify wellness-related needs in their specific population, it may be advantageous to repeat the needs assessment periodically to address changes between academic cohorts.

Acknowledgements: The authors would like to acknowledge Sylvia Wasson (2019 PharmD Candidate), Zoë Biggart (2019 PharmD Candidate), Kelly Hicks, Brenda Walton, and Dr. Jennifer Lamberts for their assistance in facilitating the exercise and nutrition classes.

\section{Conflict of Interest: None}

Funding/Support: This study was funded in part by the Ferris State University College of Pharmacy Alumni Board of Directors.

\section{References}

1. Mazurek Melnyk B, Slevin C, Militello L, et al. Physical health, lifestyle beliefs and behaviors, and mental health of entering graduate health professional students: Evidence to support screening and early intervention. J Am Assoc Nurse Pract. 2016;28(4):204-11. DOI: 10.1002/2327-6924.12350.

2. Zvauya R, Oyebode F, Day EJ, et al. A comparison of stress levels, coping styles and psychological morbidity between graduate-entry and traditional undergraduate medical students during the first 2 years at a UK medical school. BMC Res Notes. 2017;10(1):93. DOI: 10.1186/s13104-017-2395-1.

3. Stress management: Approaches for preventing and reducing stress. Boston (MA): Harvard Health Publications; 2013.

4. Compton MT, Carrera J, Frank E. Stress and depressive symptoms/dysphoria among US medical students: results from a large, nationally representative survey. J Nerv Ment Dis. 2008; 196(12):891-7. DOI: 10.1097/NMD.0b013e3181924d03.

5. McGrady A, Brennan J, Lynch D, et al. A wellness program for first year medical students. Appl Psychophysiol Biofeedback. 2012;37(4):253-60. DOI: 10.1007/s10484-012-9198-x.

6. Shapiro S, Schwartz G, Bonner G. Effects of mindfulness- based stress reduction on medical and premedical students. J Behav Med. 1998 Dec;21(6):581-99.

7. El Ansari W, Suominen S, Draper S. Correlates of achieving the guidelines of four forms of physical activity, and the relationship between guidelines achievement and academic performance: undergraduate students in Finland. Cent Eur J Public Health. 2017;25(2):87-95. DOI:

10.21101/cejph.a4387.

8. Cohen S, Kamarck T, Mermelstein R. A global measure of perceived stress. J Health Soc Behav. 1983 Dec;24(4):385-96. DOI: 10.2307/2136404.

9. Robroek SJW, van Lenth FJ, van Empelen P, et al. Determinants of participation in worksite health promotion programmes: A systematic review. Int J Behav Nutr Phys Act. 2009 May 20;6:26. DOI: 10.1186/1479-5868-6-26. 